\title{
Efeito da hiperprolactinemia induzida pela metoclopramida na córnea de camundongas
}

\section{Effectsof metoclopramide-induced hyperprolactinemia on the murinecorneal}

\author{
Carina Verna ${ }^{1}$ \\ José Maria Soares Júnior ${ }^{2}$ \\ Fernanda Watanabe Martins ${ }^{3}$ \\ Regina Célia Teixeira ${ }^{4}$ \\ Rejane Mosquette $^{5}$ \\ RicardoSantos Simões ${ }^{6}$ \\ Manuel de Jesus Simões ${ }^{7}$ \\ Edmund Chada Baracat ${ }^{8}$
}

Trabalho realizado na Universidade Federal de São Paulo - UNIFESP, Departamento de Morfologia, Disciplina de Histologia e Biologia Estrutura.

Pós-graduanda do Departamento de Ginecologia da Universidade Federal de São Paulo - UNIFESP - São Paulo (SP) - Brasil.

2 Professor Doutor do Departamento de Ginecologia da UNIFESP - São Paulo (SP) - Brasil.

${ }^{3}$ Tecnóloga Oftálmica da UNIFESP - São Paulo (SP) Brasil.

${ }^{4}$ Pós-graduanda do Departamento de Morfologia da UNIFESP - São Paulo (SP) - Brasil.

${ }^{5}$ Pós-graduanda do Departamento de Morfologia da UNIFESP - São Paulo (SP) - Brasil.

${ }^{6}$ Residente do Departamento de Ginecologia da UNIFESP - São Paulo (SP) - Brasil.

${ }^{7}$ Prof. Associado da Disciplina de Histologia da UNIFESP - São Paulo (SP) - Brasil.

${ }^{8}$ Professor Titular do Departamento de Ginecologia da UNIFESP - São Paulo (SP) - Brasil.

Endereço para correspondência: José Maria Soares Júnior. Rua Sena Madureira, 1245 - Apto. 11 - São Paulo (SP) CEP 04021-051

E-mail: jsoares415@hotmail.com

Recebido para publicação em 09.11.2005

Última versão recebida 19.12.2005

Aprovação em 16.01.2006

Projeto aprovado e financiado pela FAPESP no $03 / 03420-9$

\section{RESUMO}

Objetivo: Avaliar as alterações morfológicas promovidas pela hiperprolactinemia induzida pela metoclopramida na córnea de camundongas durante a fase de proestro e na gestação. Métodos: Quarenta camundongas adultas foram divididas, aleatoriamente, em dois grupos, a saber: controle (CTR1) e metoclopramida (MET1). Após 50 dias metade dos animais de cada grupo foram sacrificados. O restante dos animais foi acasalado, constituindo dois grupos: controle prenhe (CTR2) e o metoclopramida prenhe (MET2), que foi sacrificado no 6으 dia de gestação. Após decapitação dos animais coletou-se sangue para dosagens de estradiol, progesterona e prolactina, em seguida removidos os globos oculares para estudo histomorfométrico da córnea. Resultados: As espessuras do epitélio, estroma, endotélio e a espessura total das córneas dos grupos experimentais: MET1 e MET2 mostraram-se mais espessados quando comparados com os grupos controles: CTR1 e CTR2, respectivamente. Houve redução dos níveis hormonais do estrogênio e da progesterona nos animais que receberam metoclopramida em comparação com os respectivos controles (CTR1: estradiol $=156,6 \pm 42,2 \mathrm{pg} / \mathrm{ml}$; progesterona $=39,4 \pm 5,1 \mathrm{ng} / \mathrm{ml}$; prolactina $=130,4 \pm 26,2 \mathrm{ng} / \mathrm{ml} ;$ MET1: estradiol $=$ $108,0 \pm 33,1 \mathrm{pg} / \mathrm{ml}$; progesterona $=28,0 \pm 6,4 \mathrm{ng} / \mathrm{ml}$; prolactina $=551,5 \pm$ $23,3 \mathrm{ng} / \mathrm{ml}$; CTR2: estradiol $=354,0 \pm 56,0 \mathrm{pg} / \mathrm{ml}$; progesterona $=251,0 \pm$ $56,0 \mathrm{ng} / \mathrm{ml}$; prolactina $=423,2 \pm 28,1 \mathrm{ng} / \mathrm{ml} ;$ MET2: estradiol $=293,0 \pm$ $43,0 \mathrm{pg} / \mathrm{ml}$; progesterona $=184,0 \pm 33,0 \mathrm{ng} / \mathrm{ml}$; prolactina $=823,1 \pm 51,1 \mathrm{ng} / \mathrm{ml}$ ). Conclusão: A hiperprolactinemia induzida pela metoclopramida produziu espessamento da córnea, sobretudo, em camundongas prenhes. Possivelmente este efeito está relacionado com a redução da produção hormonal de estrogênio e de progesterona.

Descritores: Hiperprolactinemia/induzido quimicamente; Metoclopramida/administração \& dosagem; Córnea/patologia; Histocitometria; Ratos

\section{INTRODUÇÃO}

É relatado que em algumas mulheres com glaucoma, as pressões intraocular sistêmica e o peso aumentaram durante o período para-menstrual, que compreende quatro dias precedentes a menstruação e quatro dias da menstruação. Estes dados sugerem relação entre os hormônios sexuais e a produção de humor aquoso pelo olho ${ }^{(1)}$. Além disso, foi detectado maior concentração de prolactina no humor aquoso do que na circulação em mulheres normais, que pode sugerir que a prolactina teria também papel na regulação da fisiologia das células banhadas pelo humor aquoso. Estes autores relacionaram ainda a prolactina com a gênese do glaucoma agudo ${ }^{(2)}$. 
A prolactina (PRL) é um hormônio polipeptídico secretado pela hipófise. É constituída por uma cadeia de 198 aminoácidos, com peso molecular de 23 quilodaltons ${ }^{(3)}$. A detecção na espécie humana só foi possível após o advento da técnica de radioimunoensaio $^{(4)}$. É conhecida por controlar a secreção láctea, bem como, exerce ação sistêmica, incluindo seu efeito na reprodução tanto em seres humanos quanto em outros mamíferos ${ }^{(5)}$.

A prolactina atua de modo sinérgico com a progesterona e o estrogênio na promoção do crescimento e do funcionamento das glândulas mamárias e outros tecidos-alvo. Está também envolvida com o equilíbrio hídrico, bem como no crescimento e diferenciação celular, regulação da síntese de proteínas, e regulação de respostas imunológicas ${ }^{(6-7)}$.

$\mathrm{Na}$ área experimental, a metoclopramida tem sido bastante utilizada em animais, com o propósito de estudar os níveis séricos de PRL e os efeitos da elevação deste hormônio ${ }^{(5)}$, por atuar diretamente sobre a hipófise, aumentando a síntese de prolactina $^{(8)}$. Nas glândulas lacrimais de camundongas adultas, a hiperprolactinemia induzida por esse fármaco induz a redução do trofismo desse tecido ${ }^{(9)}$.

Em humanos, contribui com a regulação hormonal da pressão intra-ocular. Este fato se baseia em estudos que compararam a medida da pressão intra-ocular de pacientes com hiperprolactinemia e voluntários saudáveis, mostrando aumento de aproximadamente $38 \%$ maior em participantes com hiperprolactinemia do que em os sem essa alteração endócrina ${ }^{(10)}$. Há relatos sobre aumento de pressão intra-ocular, pressão sangüínea e peso, simultaneamente, durante o período de prémenstruação. Além disso, esta elevação é maior nas pacientes com glaucoma de ângulo fechado do que aquelas com glaucoma crônico simples. Estes fatos reforçam a relação da prolactina e a produção de humor aquoso ${ }^{(2)}$. Estudos recentes mostram que pacientes com hipertensão ocular têm a espessura da córnea central mais espessa do que pessoas normais. Essas mudanças endoteliais estão possivelmente relacionadas com a elevação crônica da pressão intra-ocular ${ }^{(11)}$. Já pacientes com glaucoma com estabilização da pressão intra-ocular, apresentaram córneas mais finas do que as normais ${ }^{(12)}$.

A interação entre a prolactina e o cortisol é capaz de marcar um notável aumento na produção de humor aquoso ${ }^{(13)}$. No epitélio do corpo ciliar, a prolactina poderia modular positivamente a ação dos corticóides, elevando a pressão intra-ocular. Além disto, detectou-se maior concentração de prolactina no humor aquoso do que na circulação, que pode sugerir que a prolactina teria também papel na regulação da fisiologia das células banhadas pelo humor aquoso ${ }^{(13)}$. Contudo os dados sobre os efeitos da hiperprolactinemia na córnea ainda são escassos. Portanto, o presente trabalho avaliou a histomorfologia e histomorfometria da córnea de camundongas tratadas com metoclopramida durante a fase de proestro (pico hormonal de esteróides sexuais) e a gestação (elevação de progesterona).

\section{MÉTODOS}

O estudo foi realizado no Biotério Central do Departamento de Morfologia, Disciplina de Histologia e Biologia Estrutural e aprovada pelo Comitê de Ética e Pesquisas da Universidade Federal de São Paulo - Escola Paulista de Medicina (UNIFESPEPM). Foram utilizadas camundongas (Mus musculus) adultas, albinas, com 100 dias de idade, virgens, procedentes do Centro de Desenvolvimento de Modelos de Experimentação (CEDEME) da Universidade Federal de São Paulo. Os animais foram mantidos, em número de 10 por gaiola, com alimentação e água ad libitum, em temperatura ambiente de $22^{\circ} \mathrm{C}$, iluminação artificial com lâmpadas fluorescentes marca Phillips (modelo luz do dia de $40 \mathrm{~W}$ ). Foram mantidos em fotoperíodo claro de 12 horas intercalado com período escuro de 12 horas, sendo o período de luz das 6:00 às 18:00 horas.

Após uma semana de adaptação ao novo ambiente, foram colhidos esfregaços vaginais diários durante 15 dias consecutivos para monitorar a periodicidade do ciclo estral. O material citológico obtido por esfregaço vaginal foi colocado em lâminas histológicas e fixado em solução álcool-éter em partes iguais, sendo então submetido à coloração de Harris-Shorr. Assim foram determinadas as fases do ciclo estral, ou seja, o proestro, estro, metaestro e diestro. Somente os animais que apresentavam dois ciclos estrais normais (4 a 5 dias) foram incluídos no estudo.

Quarenta animais foram divididos, aleatoriamente, inicialmente em dois grupos: controle não-prenhe (CTR1) - composto de 20 camundongas que receberam injeções diárias, via subcutânea, de $0,2 \mathrm{ml}$ de solução salina a $0,9 \%$, e metoclopramida não-prenhe (MET1) - constituído de 20 camundongas que receberam injeções diárias, via subcutânea, de $200 \mu \mathrm{g}$ de metoclopramida, dissolvidos em $0,2 \mathrm{ml}$ de solução salina a $0,9 \%$. A dose e a duração de tratamento com metoclopramida que induz hiperprolactinemia foi determinada em outro experimento realizado em nosso laboratório ${ }^{(5)}$.

Todos os animais receberam solução salina ou metoclopramida durante 50 dias consecutivos, sempre às 18:00 horas. Após a administração do fármaco ou da solução fisiológica, colhia-se material para avaliar o ciclo estral. No $50^{\circ}$ dia, logo após a administração das soluções, os animais de ambos os grupos que se encontravam na fase de proestro foram sacrificados. Os animais restantes foram então colocados para acasalamento, na proporção de três fêmeas para cada macho, sabidamente reprodutor.

Comprovou-se a cópula pela presença de tampão de espermatozóides na vagina. Em alguns animais, que apresentavam dilatação do orifício externo da vagina, realizou-se a coleta do material presente no seu interior para identificação de espermatozóides em microscopia de luz. Metade dos animais prenhe não foi tratada, constituindo o grupo controle prenhe (CRT2), e a outra metade foi medicada com metoclopramida, como descrito anteriormente, constituindo então o grupo metoclopramida prenhe (MET2). Sacrificaram-se os animais destes dois grupos no 6 을 dia de prenhez.

Imediatamente após a decapitação dos animais, coletou-se sangue para determinação hormonal (estrogênio, progesterona e prolactina) e a seguir, retiraram-se os globos oculares para processamento histológico e estudo em microscopia de 
luz. Para tanto, primeiramente, mergulhou-se os globos oculares em formol a $10 \%$ tamponado, durante duas horas, posteriormente as córneas foram retiradas com auxilio de tesoura e cortadas ao meio. Em seguida foram mergulhadas novamente em formol e após 10 horas processadas para inclusão em parafina. Na sequiência, incluíram-se as peças histológicas de tal maneira que pudéssemos observar cortes transversais da porção média da córnea ao microscópio de luz. Os blocos de parafina foram cortados em micrótomo do tipo Minot, ajustado para $3 \mu \mathrm{m}$ e os cortes obtidos foram corados pela hematoxilina e eosina (H.E). Parte do soro congelado a $-20^{\circ} \mathrm{C}$ foi enviado para a Califórnia (EUA) para dosagem da prolactina.

\section{Avaliação morfométrica}

Realizou-se a avaliação histológica e morfométrica por captura de imagens, as quais foram analisadas pelo programa AxionVision da Zeiss. A captura de imagens consiste em um microscópio de luz Axiolab Standart, acoplado a uma câmera de vídeo de alta resolução AxionCam (Carl Zeiss do Brasil Ltda), que transmite a imagem a um computador que contém este programa.

Para cada animal, foram feitas 5 lâminas e em cada uma, três leituras, uma na área central e outras duas na porção periférica, sempre tomando o cuidado de se considerar a menor espessura observada. Para cada animal foi efetuado um valor médio dessas 15 medidas. Analisou-se, em cada lâmina de córnea, os seguintes itens: 1) espessura de epitélio anterior; 2) espessura de estroma; 3) espessura de endotélio e; 4) espessura total da córnea.

\section{Determinação sérica de estradiol, progesterona e prolactina}

No sangue obtido foram dosados por imunoensaio enzimático quimioluminescente competitivo em fase sólida, os níveis de estradiol e de progesterona (Immulite ${ }^{\circledR}$, USA), com sensibilidade de $15 \mathrm{pg} / \mathrm{ml}$ e $0,2 \mathrm{ng} / \mathrm{ml}$ respectivamente para o estradiol e a progesterona, com alta especificidade para estes hormônios.

Os níveis séricos de prolactina foram determinados por radioimunoensaio homólogo, usando "mouse prolactin RIA kit". Procedimento realizado no "Pituitary hormones and antisera Center Harbor - UCLA Medical Center, Califórnia”.

Analisaram-se os resultados pelo teste t de Student não pareado separando-se os animais não-prenhes das prenhes. Levando-se em consideração os resultados dos animais com e sem tratamento pela metoclopramida durante a fase de proestro (CTR1 e MET1), em separado das camundongas prenhes (CTR2 e MET2).

\section{RESULTADOS}

\section{Morfológicos}

Grupo controle não-prenhe (CTR1): a córnea apresentouse mais delgada em relação ao grupo que recebeu metoclopramida: na região anterior nota-se epitélio estratificado pavimentoso não queratinizado constituído por 5 a 6 camadas de células, o estroma está formado por lamelas colágenas e na região poste- rior da córnea nota-se a presença de um epitélio pavimentoso simples, denominado de endotélio posterior. Observou-se em algumas regiões a presença de eosinófilos (Figura 1A).

Grupo metoclopramida não prenhe (MET1): a córnea apresenta a mesma arquitetura observada no grupo controle não prenhe em relação ao epitélio anterior, estroma e endotélio posterior. Nota-se que o epitélio anterior da córnea está constituído por 9 a 10 camadas de células, o estroma mais espesso, com maior número de lamelas colágenas, quando comparado ao grupo controle não-prenhe. No endotélio posterior da córnea não se notou alterações (Figura 1B).

Grupo controle prenhe (CTR2): a córnea apresentou-se parecida ao do grupo controle não prenhe, ou seja, mais delgada. O epitélio anterior da córnea está constituído por 6 a 7 camadas de células; o estroma formado por tecido conjuntivo denso, e o endotélio posterior muito parecido ao do controle não prenhe (CTR1). Observou-se eosinófilos nas bordas da córnea (Figura 1C).

Grupo metoclopramida prenhe (MET2): As córneas deste grupo apresentaram-se bem parecidas com as do grupo metoclopramida não-prenhe. Notamos que o epitélio anterior apresenta-se constituído por 7-8 camadas de células, sendo o estroma mais espesso, com maior número de lamelas colágenas, quando comparado ao grupo controle prenhe. No endotélio posterior da córnea não notamos alterações (Figura 1D).

\section{Morfométricos}

A espessura do epitélio, estroma, endotélio e o total da córnea dos grupos não-prenhe (MET1) e prenhe (MET2) que receberam metoclopramida mostraram-se significativamente aumentados em relação aos respectivos grupos controle, nãoprenhe (CTR1) como o prenhe (CTR2), que não receberam metoclopramida. Os valores das espessuras do epitélio, estroma, endotélio e a espessura total das córneas dos grupos experimentais: MET1 $(10,6 \pm 0,6 ; 54,7 \pm 3,5 ; 3,7 \pm 1,2 ; 98,8 \pm$

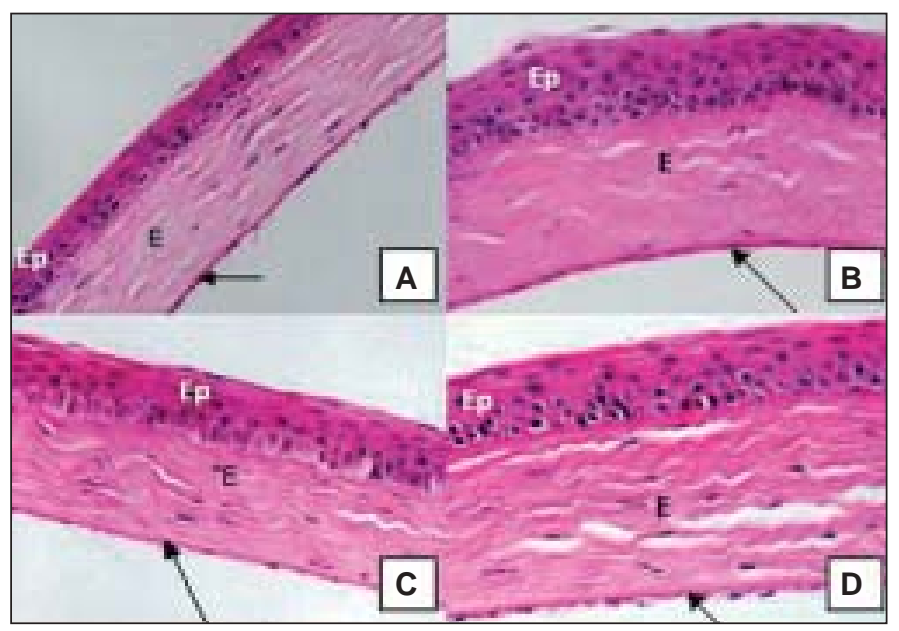

Figura 1 - Fotomicrografias mostrando parte dos cortes das córneas das camundongas pertencentes ao grupo CTR1 (A), MET1 (B), CRT2 (C)

e MET (D). Observar epitélio anterior da córnea (Ep), estroma (E) e endotélio posterior da córnea (seta). H.E.= aumento de 200x. 
$5,4 \mu \mathrm{m})$ е MET2 $(9,7 \pm 0,7 ; 62,5 \pm 4,4 ; 3,5 \pm 1,2 ; 102,9 \pm 6,7 \mu \mathrm{m})$ mostraram-se mais espessados quando comparados com os grupos controles: CTR1 $(7,6 \pm 0,5 ; 60,8 \pm 3,9 ; 3,8 \pm 1,0 ; 103,8 \pm$ $5,5 \mu \mathrm{m})$ e CTR2 $(7,6 \pm 0,5 ; 47,5 \pm 2,8 ; 3,2 \pm 0,4 ; 79,7 \pm 3,8 \mu \mathrm{m})$, respectivamente (Tabela 1 ).

\section{Dosagens de estradiol e progesterona}

Houve aumento significativo nos níveis de estrogênio e progesterona nos grupos prenhes (CTR2 e MET2) em comparação aos não-prenhes (CTR1 e MET1) ( $\mathrm{p}<0,05)$. Quando comparado os grupos com e sem metoclopramida, detectou-se redução significante nos valores desses hormônios nos grupos não-prenhe (MET1) e prenhe (MET2) que receberam metoclopramida, em relação aos grupos não prenhe (CTR1) e prenhe (CTR2) que receberam apenas veículo, respectivamente $(\mathrm{p}<0,05)$. Os níveis de prolactina mostraram-se aumentados nos grupos MET1 e MET2, quando comparados com os grupos CTR1 e CTR2 respectivamente (Tabela 2).

\section{DISCUSS ÃO}

Os primeiros estudos sobre a ação de hiperprolactinemia sobre o globo ocular foram em $1987^{(8)}$. Estes autores mostraram que a hiperprolactinemia aumenta a pressão intra-ocular humana. Entretanto, pouco se sabe sobre as repercussões deste aumento pressórico sobre as estruturas do globo ocular ${ }^{(10)}$.

Alguns trabalhos podem auxiliar na hipótese de que a hiperprolactinemia alterando a pressão intra-ocular, determine alterações morfológicas no olho. Reforçando esta idéia, estudos mostram correlação entre a espessura da córnea e a pressão intra-ocular ${ }^{(14)}$. Estudo com perimetria computadorizada e paquimetria ultra-sônica em pacientes com pressão intra-ocular elevada com disco óptico normal mostraram que a espessu- ra corneal central dos pacientes com anormalidades de campo visual foi significantemente mais baixa, que a espessura corneal central dos pacientes com hipertensão intra-ocular com campo visual normal. Neste estudo, os autores evidenciaram que pacientes com hipertensão intra-ocular e perda de campo visual apresentaram redução significante da espessura da córnea central em relação aos com hipertensão intra-ocular e campo visual normal. Assim, estes autores sugerem que a espessura da córnea central deveria ser observada para o acompanhamento de pacientes com elevação da pressão intra-ocular com risco de desenvolvimento de danos glaucomatosos ${ }^{(14)}$.

Assim, a espessura aumentada da córnea das camundongas que receberam metoclopramida poderia estar associada com elevação da pressão intra-ocular nesses animais. Contudo, esta hipótese deverá ser investigada em futuros experimentos para investigar se o efeito da elevação da prolactina sérica atua de modo direto ou indireto nos órgãos em estudo.

\section{CONCLUSÃO}

Nosso estudo mostra que a hiperprolactinemia induzida pela metoclopramida aumenta as camadas da córnea, principalmente em camundongas prenhe e esta alteração poderia estar relacionada com redução dos hormônios sexuais circulantes ou com a ação da prolactina sérica.

\section{AGRADECIMENTOS}

Os autores agradecem ao Prof. Dr. A. F. Parlow, Diretor Científico do National Hormone \& Pituitary Program HarborUCLA Medical Center pelo interesse no nosso trabalho e pela dosagem sérica de prolactina.

\begin{tabular}{|lcccccc|}
\hline \multicolumn{7}{|l}{ Tabela 1. Efeitos do tratamento com metoclopramida sobre os resultados das análises morfológicas e morfométricas na córnea em } \\
camundongas prenhes e não-prenhes
\end{tabular}

\begin{tabular}{|c|c|c|c|c|c|}
\hline Estado fisiológico & Tratamento & Grupo & Estradiol $(\mathrm{pg} / \mathrm{ml})$ & Progesterona (ng/ml) & Prolactina (ng/ml) \\
\hline Não-prenhe & $\begin{array}{l}\text { Veículo } \\
\text { Metoclopramida }\end{array}$ & $\begin{array}{l}\text { CTR1 } \\
\text { MET1 }\end{array}$ & $\begin{array}{l}156,6 \pm 42,2 \\
108,0 \pm 33,1^{a}\end{array}$ & $\begin{array}{l}39,4 \pm 5,1 \\
28,0 \pm 6,4^{a}\end{array}$ & $\begin{array}{l}130,4 \pm 26,2 \\
551,5 \pm 23,3^{a}\end{array}$ \\
\hline
\end{tabular}




\section{ABSTRACT}

Purpose: To evaluate the morphological changes in murine cornea upon metoclopramide-induced hyperprolactinemia during the proestrous phase or pregnancy. Methods: Forty adult mice were divided into two groups: (control) CTR1 and (treated with metoclopramide (MET1). After fifty days, half of the mice were sacrificed. The remaining animals were mated, and then labeled as pregnant controls (CTR2). Part of these animals were treated with metoclopramide and constituted the metoclopramide-treated pregnant (MET2) group. The groups CTR2 and MET2 were sacrificed on the 6th day of pregnancy. The hormonal levels were assessed by chemioluminescence and radioimmunoassay methods and the cornea was removed for the histomorphometric study. Results: The epithelial, stromal, endothelial and total thickness in the experimental group was: MET1 and MET2 were higher than one in the control group: CTR1 and CTR2. There was a significant reduction of the hormonal level in the animals that received metoclopramide as compared to controls (CTR1: estradiol $=156.6 \pm 42.2 \mathrm{pg} / \mathrm{ml}$; progesterone $=$ $39.4 \pm 5.1 \mathrm{ng} / \mathrm{ml} ;$ prolactin $=130.4 \pm 26.2 \mathrm{ng} / \mathrm{ml} ;$ MET1: estradiol $=$ $108.0 \pm 33.1 \mathrm{pg} / \mathrm{ml} ;$ progesterone $=28.0 \pm 6.4 \mathrm{ng} / \mathrm{ml} ;$ prolactin $=$ $551.5 \pm 23.3 \mathrm{ng} / \mathrm{ml}$; CTR2: estradiol $=354.0 \pm 56.0 \mathrm{pg} / \mathrm{ml}$; progesterone $=251.0 \pm 56.0 \mathrm{ng} / \mathrm{ml}$; prolactin $=423.2 \pm 28.1 \mathrm{ng} / \mathrm{ml} ;$ MET2: estradiol $=293.0 \pm 43.0 \mathrm{pg} / \mathrm{ml}$; progesterone $=184.0 \pm 33.0 \mathrm{ng} / \mathrm{ml}$; prolactin $=823.1 \pm 51.1 \mathrm{ng} / \mathrm{ml})$. Conclusion: The metoclopramide-induced hyperprolactinemia may increase corneal layers, mainly in pregnant mice. Possibly, this effect is related to reduction in estrogen and progesterone production.

Keywords: Hyperprolactinemia/chemically induced; Metoclopramide/administration \& dosage; Cornea/pathology; Histocytochemistry; Mice

\section{REFERÊNCIAS}

1. Dalton K. Influence of menstruation on glaucoma. Br J Ophthalmol. 1967;51 (10):692-5.

2. Pinard CL, Weiss ML, Brightman AH, Fenwick BW, Davidson HJ. Evaluation of lysozyme and lactoferrin in lacrimal and other ocular glands of bison and cattle and in tears of bison. Am J Vet Res. 2003;64(1):104-8.

3. Meuleman EJ, van Lankveld JJ. Hypoactive sexual desire disorder: an underestimated condition in men. BJU Int. 2005;95(3):291-6.

4. Lewis UJ, Singh RN, Seavey BK. Human prolactin: isolation and some properties. Biochem Biophys Res Commun. 1971;44(5):1169-76.

5. Rossi AG, Soares JM Jr, Motta EL, Simões MJ, Oliveira-Filho RM, Haidar MA, et al. Metoclopramide-induced hyperprolactinemia affects mouse endometrial morphology. Gynecol Obstet Invest. 2002;54(4):185-90.

6. Brand JM, Frohn C, Cziupka K, Brockmann C, Kirchner H, Luhm J. Prolactin triggers pro-inflammatory immune responses in peripheral immune cells. Eur Cytokine Netw. 2004;15(2):99-104.

7. Carreno PC, Sacedon R, Jimenez E, Vicente A, Zapata AG. Prolactin affects both survival and differentiation of T-cell progenitors. J Neuroimmunol. 2005; 160(1-2):135-45.

8. Betzold CM. Galactagogues. J Midwifery Womens Health. 2004;49(2):151-4. Comment in: J Midwifery Womens Health. 2004;49(5):461; author reply 461.

9. Verna C, Martins FW, Mosquette R, Teixeira RC, Simões RS, Simões MJ, et al. Efeito da hiperprolactinemia induzida pela metoclopramida na glândula lacrimal de camundongas: Estudo experimental. Rev Bras Ginec Obstet. 2005;27 (9):524-8.

10. Warren, DW. Hormonal influences on the lacrimal gland. Int Ophthalmol Clin. 1994;34(1):19-25.

11. Shields MB. Textbook of glaucoma. Baltimore: Williams \& Wilkins; 1992.

12. Ventura AC, Bohnke M, Mojon DS. Central corneal thickness measurements in patients with normal tension glaucoma, primary open angle glaucoma, pseudoexfoliation glaucoma, or ocular hypertension. Br J Ophthalmol. 2001;85(7): $792-5$.

13. Ishimaru N, Saegusa K, Yanagi K, Haneji N, Saito I, Hayashi Y. Estrogen deficiency accelerates autoimmune exocrinopathy in murine Sjogren's syndrome through fas-mediated apoptosis. Am J Pathol. 1999;155(1):173-81.

14. Medeiros FA, Sample PA, Weinreb RN. Corneal thickness measurements and visual function abnormalities in ocular hypertensive patients. Am J Ophthalmol. 2003;135(2):131-7. Comment in: Am J Ophthalmol. 2003;136(5):964-5; author reply 965-6. 